\title{
The Inflection Point of the Speed-Density Relation and the Social Force Model
}

\author{
Tobias Kretz · Jochen Lohmiller · Johannes Schlaich \\ PTV Group, Karlsruhe, Germany, \\ E-mail: tobias.kretz@ptvgroup.com
}

Received: 26 May 2016 / Accepted: 24 October 2016

DOI: $10.17815 / \mathrm{CD} .2016 .6$

\begin{abstract}
It has been argued that the speed-density diagram of pedestrian movement has an inflection point [1] (p. 3, "Domain I: ... At low densities there is a small and increasing decline of the velocity ... Domain III: ... For growing density the velocity remains nearly constant."). This inflection point was found empirically in investigations of closed-loop single-file pedestrian movement.

The reduced complexity of single-file movement does not only allow a higher precision for the evaluation of empirical data, but it also significantly simplifies analytical considerations. This is especially true if one assumes homogeneous conditions, i.e. neglects temporal variations (consider time averages, neglect stop-and-go waves), individual differences of pedestrians (all simulated pedestrians have identical parameters) and investigates only steady-state (not the initial phase). As will be shown in this contribution one then can make a transition from the microscopic to a continuous and macroscopic perspective.

Building on that it will be shown that certain (common) variants of the Social Force Model (SFM) do not produce an inflection point in the speed-density diagram if - assuming periodic boundary conditions - infinitely many pedestrians contribute to the force computed for one pedestrian. It will furthermore be shown that if - in said $1 \mathrm{~d}$ movement situation - one only considers nearest neighbors for the computation of the interpedestrian forces the Social Force Model in the continuous description results in the so called Kladek formula for the speed-density relation. Since the Kladek formula exhibits the desired inflection point this observation is used as a motivation for an extension of the Social Force Model which allows to transform the continuous description of the SFM continuously to the Kladek formula and which also exhibits the inflection point in the speed density relation. It will be shown then, that this extended SFM yields astonishingly similar speed density relations as the original SFM when only a fixed limited number of (nearest) pedestrians are considered in the computation of the inter-pedestrian force.
\end{abstract}


Finally it will be discussed, if also the description of the speed-density diagram for (motorized, four-wheel) vehicular and/or bicycle traffic could benefit from these measures.

Keywords Pedestrians $\cdot$ speed density relation $\cdot$ Social Force Model $\cdot$ Kladek Formula

\section{Introduction I: Empirical Data on Pedestrians' Speed-Density Relation}

In the course of recent years a number of experiments have been conducted in which pedestrians walk single-file in a closed loop [1-5]. Having a different number of pedestrians in the loop different densities are prepared. In a section of the loop density - in this case: line density [persons / m] - and speed are measured. Fig. 1 shows the experimental setup from which most data stems. There were experiment runs in which the loop was larger and more pedestrians participated, but the principle was always the same.
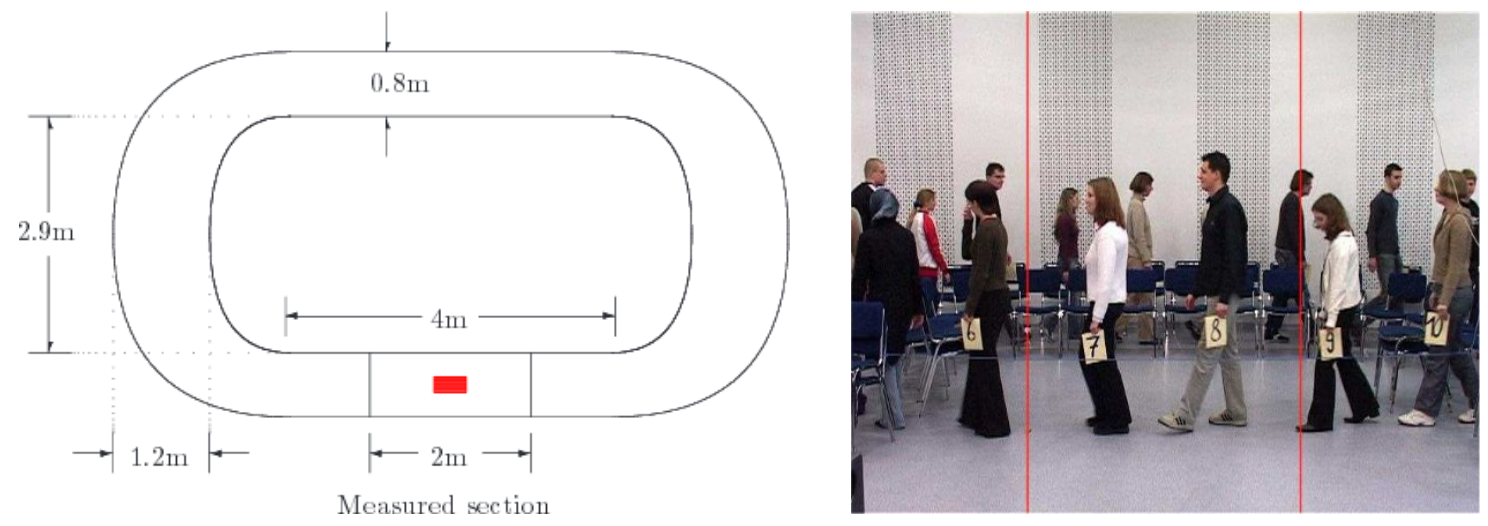

Figure 1 Experimental setup. Source: Figures 2 and 3 of [1]

The experiment has been conducted at various places around the world. Fig. 2 shows the results for India and Germany. With the free speeds plotted in this diagram the existence of an inflection point and even more so a positive curvature at high densities is obvious, although the density of the inflection point can only be estimated roughly.

Whereas Fig. 2 clearly shows that differences between India and Germany were found, the loop size (corridor length) and the profession of participants did not make a difference, at least not an obvious one, see Fig. 3.

Where free speeds are not shown in a diagram (e.g. Fig. 3) and data for very small densities is missing as well the existence of an inflection point may not be immediately obvious, however, assuming typical free speeds it becomes clear that it must exist, and even more important the curvature at high densities is without doubt positive. This holds independently of the measurement method, see Fig. 4.

Furthermore the discussion in section 2 of [1] - see Fig. 5 - indicates that the existence of an inflection point is common sense and even more so that for - at least moderately - high densities the curvature of the flow density relation is positive. If there is in fact a 

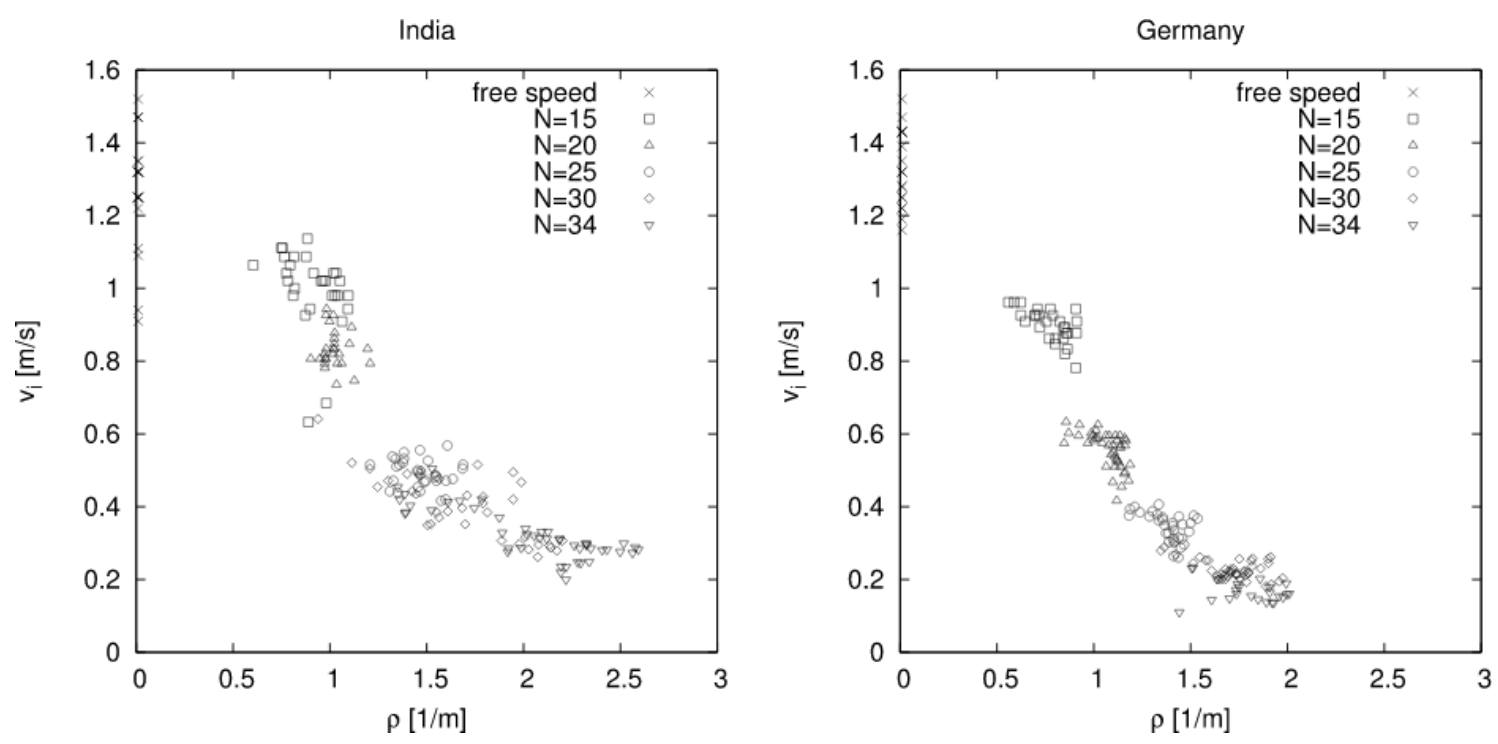

Figure 2 Speed density diagram for India (left) and Germany (right). Source: Figure 5 of [2]
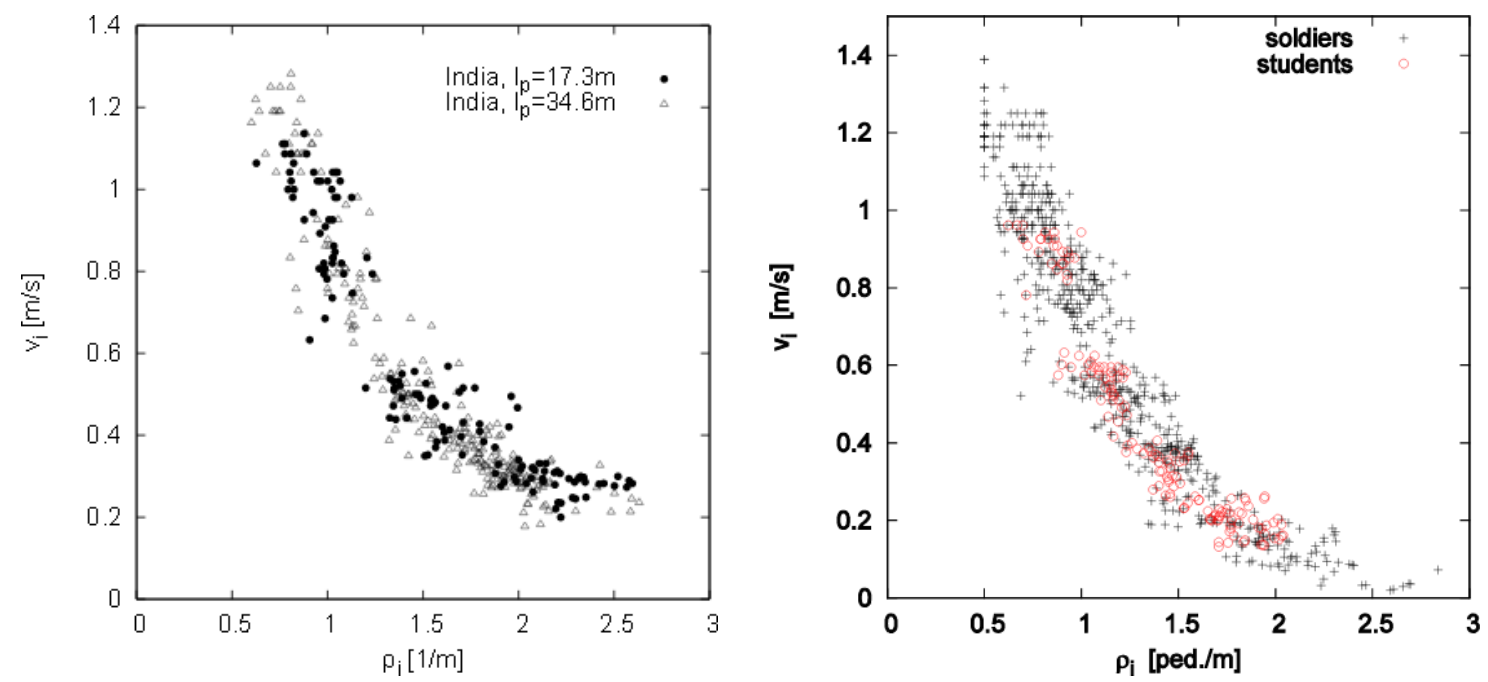

Figure 3 Speed density diagram for different loop sizes and professions in India (left) and Germany (right). In India the participants in both experiments were students, in Germany the participants in the short loop experiment were students, the participants in the long loop were soldiers. Sources: left: Figure 7 of [2]; right: Figure 1 of [5]

second inflection point at very high densities as shown in Fig. 5 or if speed approaches zero nearly asymptotically as Fig. 4 indicates will not be discussed in this contribution. Note that the speed-density diagram of Weidmann $[6,7]$ is a summary of many different measurements, including not only single-file measurement and even bi-directional flow investigations, which may have contributed to the depicted course of the average function at high densities.

Weidmann approximated the speed-density diagram with the so called Kladek formula which will be discussed in the next section. 



Figure 4 Speed density diagram created from the same data as in Fig. 3 (right, soldiers), but with different evaluation methods. On the left side the raw data is evaluated using the Voronoi method, on the right side a snap shot of all participants in the whole loop is taken at one point in time (resp. two frames) and speeds are measured whereas for density the global average density is used. Sources: left: Figure 3 of [3]; right: Figure 6 of [4]

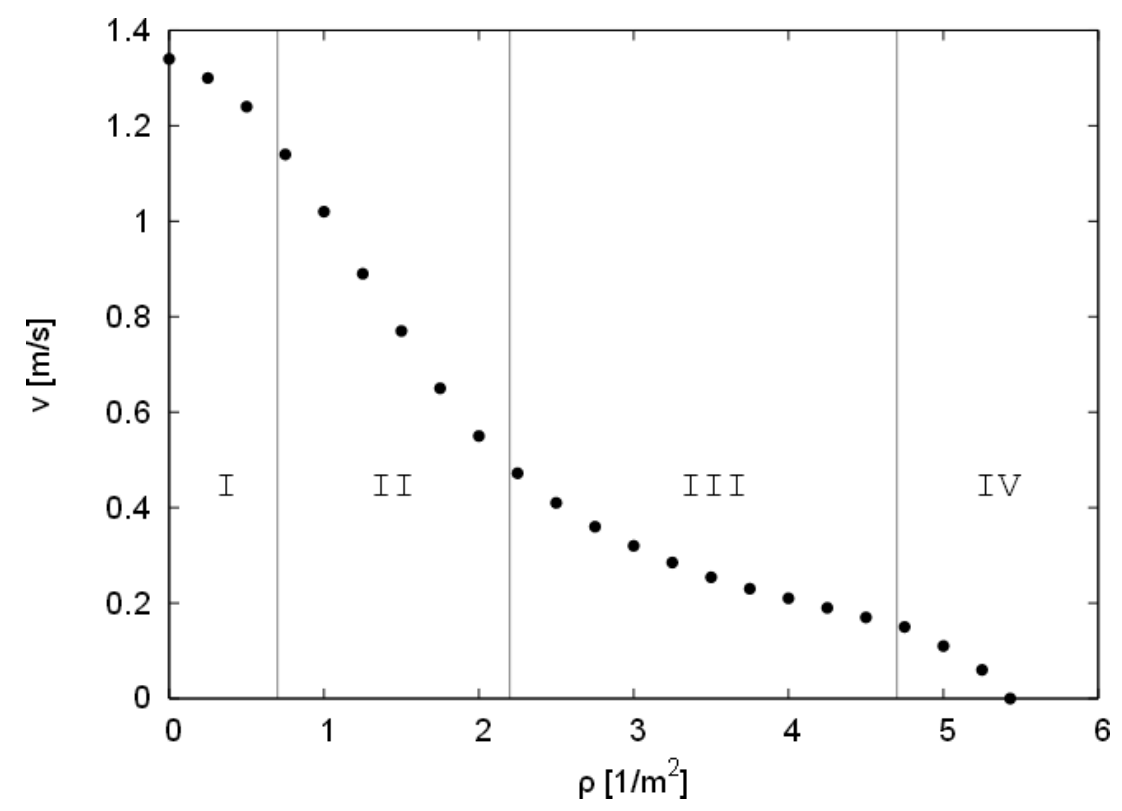

Figure 5 Fundamental diagram according to Weidmann [6,7] as given and discussed in [1].

\section{Introduction II: the Kladek Formula}

In 1966 Kladek [8] suggested the following equation as macroscopic function to describe the relation between average momentary speed $\bar{v}_{m}$ and density $\rho$ of motorized urban road traffic:

$$
\bar{v}_{m}=\bar{v}_{f}\left(1-e^{-\gamma\left(\frac{1}{\rho}-\frac{1}{\rho_{\max }}\right)}\right)
$$


with $\bar{v}_{f}$ as free speed, $\rho_{\max }$ as maximum density where speed becomes zero, and $\gamma$ a free parameter to be calibrated. The functional form was used before by Newell [9] for an optimal velocity car-following model.

If speed is expressed in fraction $f$ of the free speed $\bar{v}_{f}$ and density as fraction $x$ of the maximum (i.e. stand still) density equation (Eq. 1) reduces to

$$
f(x)=1-e^{-a\left(\frac{1}{x}-1\right)}
$$

leaving only one free parameter $-a$ - to fit the function with empirical data. The relation between parameter $a$ in Eq. 2 and parameter $\gamma$ in Eq. 1 is $\gamma=a \rho_{\max }$.

Concerning the properties of the Kladek formula, we first note that it has an inflection point which is located - for Eq. $2-$ at $x_{i}=a / 2$.

In their text book Lohse and Schnabel [10] point out that the Kladek formula has a number of favorable properties for example that one single formula covers the entire density range giving free speed for zero density and zero speed for maximum density.

It is sometimes pointed out for road traffic that the maximum of the flow is located at or near the speed drop in the speed density relation $[11,12]$. For vehicular traffic this has been achieved with piece-wise definitions of the speed-density diagram. For a smooth and analytical function as the Kladek formula this means that the inflection point of the speed-density relation - i.e. where the first derivative is most negative - has to be at the the position of the maximum of flow ${ }^{1}$. For Eq. 2 this requirement translates into two equations:

$$
\begin{aligned}
\left.\frac{\partial}{\partial x}(x f(x))\right|_{x_{c}} & =0 \\
\left.\frac{\partial^{2}}{\partial x^{2}} f(x)\right|_{x_{c}} & =0
\end{aligned}
$$

which fix the value of parameter $a$ to $a=2-\ln (3) \approx 0.901$, leaving no more freedom for calibration with empirical data. However, comparing the function with this value for $a$ as shown in Fig. 6 with the empirical data depicted in Fig. 2 to 4, suggests that this value does not yield the closest approximation to empirical data.

Fig. 6 shows that for different values of parameter $a$ the maximum in the flow-density diagram can be on the left or on the right side of $x=0.5$. Since usually in pedestrian as well as vehicular traffic the maximum is located well on the left side it is interesting to know the value of $a$ for which the maximum is exactly at $x=0.5$ and that is where $(1+2 a)=e^{a}$, so $a=-W_{-1}(-0.5 \exp (-0.5))-0.5 \approx 1.256$ with $W_{-1}()$ denoting the lower real-valued branch of the Lambert $\mathrm{W}$ function.

As mentioned previously Weidmann applied the Kladek formula to describe the speed density relation of uni-directional pedestrian flow. This led to the situation that - despite Kladek having introduced the equation to describe urban vehicular flow - today the Kladek formula is and has been applied predominantly for pedestrian dynamics and engineering [13-28] with only few literature search results related to vehicular traffic [29].

\footnotetext{
${ }^{1}$ This is necessarily fulfilled, if the Gaussian function is used to describe the relation between speed and density, compare [10].
} 

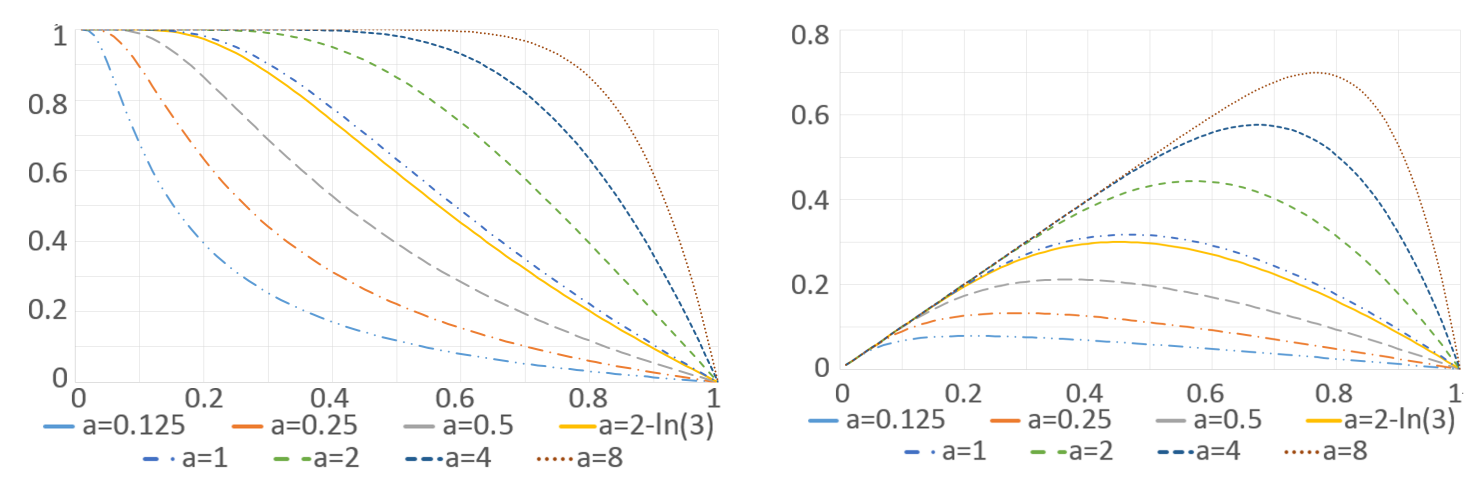

Figure 6 Speed density (left) and flow density (right) relations according to Eq. 2 for various values of parameter $a$.

One might stumble here over the fact that Kladek's system - urban road traffic - is essentially one-dimensional, while Weidmann used the very same function to describe a $2 \mathrm{~d}$ system, as Weidmann does not at all limit the applicability to single-file movement. This difference is reflected in the dimension of the density and consequently the dimension of the constant $\gamma$ in the exponent. The transfer from $1 \mathrm{~d}$ to $2 \mathrm{~d}$ is not trivial, neither for microscopic nor for macroscopic modeling and data taking, since in a $2 \mathrm{~d}$ system the longitudinal and lateral densities - i.e. measuring density on a rectangle that is extended in the direction of movement vs. measuring it on a rectangle extended orthogonal to movement - may be different and the conversion might be density-dependent itself. In this work we rest our motivation on a single-file experiment of pedestrian dynamics. The $1 \mathrm{~d}$ starting point implies that we are more "in the vein of" Kladek than Weidmann although our focus is on pedestrians. The Social Force Model was undoubtedly formulated to describe $2 \mathrm{~d}$ systems, still one would of course expect from a $2 \mathrm{~d}$ microscopic model that it is able to reproduce movement dynamics of $1 \mathrm{~d}$ situations as far as they can be prepared in reality, as the experimenters in the referred experiments have done.

Weidmann's parametrization of the Kladek formula to describe (two-dimensional) pedestrian dynamics is

$$
\begin{aligned}
v_{f} & =1.34 \mathrm{~m} / \mathrm{s} \\
\gamma & =1.9131 / \mathrm{sqm} \\
\rho_{\max } & =5.41 / \mathrm{sqm}
\end{aligned}
$$

For the dimensionless form - and therefore at least formally also applicable in a onedimensional setting - the value of parameter $a$ would be

$$
a=\frac{\gamma}{\rho_{\max }} \approx 0.354
$$

In a strict sense the fact that the Kladek formula is meant to describe average momentary speed requires for calibration first, that to measure speed it has to be measured how far pedestrians (or cars) move in a given, fixed, and very short time span, and second that each (density/speed) data point has to be an average of measurements done within 
the same time span. To put it more graphically: one has to take two pictures separated only by a very short time span and measure in both how far pedestrians or cars moved. The average of these translations divided by the time between the two pictures gives the speed and the density results from the number of cars and the length of the observed road section. To gain low-noisy data one obviously needs a large measurement section such that many entities contribute to one data point. Traffic on that section at the time of measurement has to be homogeneous and in steady-state. Clearly collecting a lot of - in the sense of these requirements - high quality data is difficult.

\section{The Social Force Model for Steady-States in Single-File Movement}

The circular specification of the Social Force Model [30] is defined $\mathrm{as}^{2}$ :

$$
\ddot{\vec{x}}_{\alpha}(t)=\frac{\vec{v}_{0 \alpha}-\dot{\vec{x}}_{\alpha}(t)}{\tau_{\alpha}}+\tilde{A}_{\alpha} \sum_{\beta} w\left(\vec{x}_{\alpha}(t), \vec{x}_{\beta}(t), \dot{\vec{x}}_{\alpha}(t), \lambda_{\alpha}\right) e^{-\frac{\left|\vec{x}_{\beta}(t)-\vec{x}_{\alpha}(t)\right|-R_{\alpha}-R_{\beta}}{B \alpha}} \hat{e}_{\alpha \beta}
$$

with

$$
w\left(\vec{x}_{\alpha}(t), \vec{x}_{\beta}(t), \dot{\vec{x}}_{\alpha}(t), \lambda_{\alpha}\right)=\lambda_{\alpha}+\left(1-\lambda_{\alpha}\right) \frac{1+\cos \left(\theta_{\alpha \beta}\left(\vec{x}_{\alpha}(t), \vec{x}_{\beta}(t), \dot{\vec{x}}_{\alpha}(t)\right)\right)}{2}
$$

where $v_{0 \alpha}$ is the desired speed of pedestrian $\alpha . \tilde{A}_{\alpha}>0, B_{\alpha}>0,0 \leq \lambda_{\alpha} \leq 1$, and $\tau_{\alpha}>0$ are parameters of the model. $R$ denotes the body radius of a pedestrian. $\hat{e}_{\alpha \beta}$ points from pedestrian $\beta$ on pedestrian $\alpha . \vec{x}$ is the position of a pedestrian and dots mark time derivatives (i.e. speed and acceleration). The sum runs over all - potentially infinitely many - pedestrians in a simulation scenario. Function $w()$ is there to suppress forces acting from behind. Within it $\theta_{\alpha \beta}$ is the angle between pedestrian $\alpha$ 's velocity vector and the line connecting pedestrians $\alpha$ and $\beta$.

From here on we assume that parameters $\tilde{A}, B, \lambda, \tau R$, and $v_{0}$ have identical value for all pedestrians, so we omit the indices. This allows to combine $\tilde{A}$ and $R$ into a new parameter:

$$
A=\tilde{A} e^{\frac{2 R}{B}}
$$

Since it is obvious which properties are time dependent, we also omit the " $(t)$ ". Then Eq. 9 can be written for the one-dimensional case:

$$
\begin{aligned}
\ddot{x}_{\alpha} & =\frac{v_{0}-\dot{x}_{\alpha}}{\tau}+A \sum_{\beta} w\left(x_{\alpha}, x_{\beta}, \lambda\right) e^{-\frac{d_{\alpha \beta}}{B}} \\
d_{\alpha \beta} & =\left|x_{\beta}-x_{\alpha}\right| \\
w\left(x_{\alpha}, x_{\beta}, \lambda\right) & =\lambda \text { if } x_{\beta}-x_{\alpha}<0 \\
w\left(x_{\alpha}, x_{\beta}, \lambda\right) & =-1 \text { if } x_{\beta}-x_{\alpha}>0
\end{aligned}
$$

\footnotetext{
${ }^{2}$ We neglect here forces from walls from the beginning since we are anyway working towards the onedimensional case.
} 
with the additional assumption that for all pedestrians and times $\dot{x}>0$. This can be done without limiting generality since we are not interested in possible oscillation phenomena far from equilibrium which might occur even if all desired speeds point to the right $\left(v_{0}>0\right)$ [31], but here we are only interested in the stationary state of the system (strong equilibrium).

Elliptical specification II is a variant of the Social Force Model where the force between pedestrians - in addition to the mutual distance - also depends on the relative velocity of pedestrians $\alpha$ and $\beta$. For the full definition we refer to [32] and here just give the simplified version parallel to the circular specification above:

$$
\begin{aligned}
\ddot{x}_{\alpha} & =\frac{v_{0}-\dot{x}_{\alpha}}{\tau}+a \sum_{\beta} w\left(x_{\alpha}, x_{\beta}, \lambda\right) \frac{\bar{d}_{a \alpha \beta}}{\bar{d}_{g \alpha \beta}} e^{-\frac{\bar{d}_{g \alpha \beta}}{b}} \\
\bar{d}_{a \alpha \beta} & =\frac{d_{\alpha \beta}+d_{f \alpha \beta}}{2} \\
\bar{d}_{g \alpha \beta} & =\sqrt{d_{\alpha \beta} d_{f \alpha \beta}} \\
d_{f \alpha \beta} & =\left|x_{\beta}-x_{\alpha}+\left(\dot{x}_{\beta}-\dot{x}_{\alpha}\right) \Delta t\right| \\
d_{\alpha \beta} & =\left|x_{\beta}-x_{\alpha}\right| \\
w\left(x_{\alpha}, x_{\beta}, \lambda\right) & =\lambda \text { if } x_{\beta}-x_{\alpha}<0 \\
w\left(x_{\alpha}, x_{\beta}, \lambda\right) & =-1 \text { if } x_{\beta}-x_{\alpha}>0
\end{aligned}
$$

with the new parameter $\Delta t$ which indicates the time to which the current evolution of distance between $\alpha$ and $\beta$ is extrapolated linearly to an expected future distance $d_{f \alpha \beta}$. $\bar{d}_{a \alpha \beta}$ and $\bar{d}_{g \alpha \beta}$ are the algorithmic and geometric mean between current and expected future distance $d_{\alpha \beta}$ and $d_{f \alpha \beta} \cdot{ }^{3}$

If - as in the scenario discussed in this work - pedestrians $\alpha$ and $\beta$ have the same velocity or if $\Delta t=0$ it is $\bar{d}_{g \alpha \beta}=\bar{d}_{a \alpha \beta}=d_{f \alpha \beta}=d_{\alpha \beta}$ and elliptical specification II gives the same force for $\alpha$ as the circular specification.

Now we investigate the steady state of this - in fact both - model(s). Steady-state means that the speeds and distances remain constant and consequently acceleration is zero for all pedestrians. With the left side of Eq. 12 being zero we can easily compute the steady state speed without having to solve a differential equation:

$$
\dot{x}_{\alpha}=v_{0}+\tau A \sum_{\beta} w\left(x_{\alpha}, x_{\beta}, \lambda\right) e^{-\frac{d_{\alpha \beta}}{B}}
$$

As we chose that the parameters for all pedestrians are equal and the system has periodic boundary conditions or is infinitely large, all distances between neighboring pedestrians are equal. Then necessarily the distance (center point to center point) from pedestrian $\alpha$ to the $n$ next neighbor $\beta_{n}$ can then be written as:

$$
d_{\alpha \beta n}=n d_{0}
$$

\footnotetext{
${ }^{3}$ There is an additional restriction: if within the time horizon $\Delta t$ according to the linear extrapolation pedestrians $\alpha$ and $\beta$ would walk through each other, the force is zero. For our further considerations this case is not relevant.
} 
If we resolve the $w()$ function into both directions $(w()=-1$ for all pedestrians ahead and $w()=\lambda$ for all pedestrians behind we can rewrite Eq. 23 more explicitly with the sum running over natural numbers not pedestrians:

$$
\dot{x}_{\alpha}=v_{0}-(1-\lambda) \tau A \sum_{n=1}^{\infty} e^{-\frac{n d_{0}}{B}}
$$

Since $d_{0}$ and $B$ both necessarily are positive it is $e^{-\frac{d_{0}}{B}}<1$ and the sum is the geometric series with the well known result

$$
\begin{aligned}
\dot{x}_{\alpha} & =v_{0}-(1-\lambda) \tau A\left(\frac{1}{1-e^{-\frac{d_{0}}{B}}}-1\right) \\
& =v_{0}-(1-\lambda) \tau A \frac{1}{e^{\frac{d_{0}}{B}}-1} \\
& =v_{0}-(1-\lambda) \tau A \frac{1}{e^{\frac{1}{B \rho}}-1}
\end{aligned}
$$

where $\rho$ is the line density of pedestrians $\rho=1 / d_{0}{ }^{4}$

With appropriately chosen values for parameters $v_{0}, \lambda, \tau A, B$ Eq. 28 should be able to reproduce the empirical speed-density diagram as shown in Fig. 2 to 4. Of course not each single data point, but as a function it should approximately match average functions computed from the empirical point clouds with an appropriate method. There is more than one such appropriate method and consequently in detail more than one average function can be extracted from a cloud of empirical data point. However, qualitative properties like the existence of an inflection point will (should) result from each such method. Therefore the speed density relation in Eq. 28 computed for/from the Social Force Model should also yield an inflection point. So we compute the second density derivative of the speed function of Eq. 28

$$
\begin{aligned}
v(\rho) & =v_{0}-(1-\lambda) \tau A \frac{1}{e^{\frac{1}{B \rho}}-1} \\
\frac{\partial v(\rho)}{\partial \rho} & =-(1-\lambda) \tau A \frac{e^{\frac{1}{B \rho}}}{B \rho^{2}\left(e^{\frac{1}{B \rho}}-1\right)^{2}} \\
\frac{\partial^{2} v(\rho)}{\partial \rho^{2}} & =(1-\lambda) \tau A e^{\frac{1}{B \rho}} \frac{(2 B \rho-1) e^{\frac{1}{B \rho}}-(2 B \rho+1)}{B^{2} \rho^{4}\left(e^{\frac{1}{B \rho}}-1\right)^{3}}
\end{aligned}
$$

and test if it is zero for some density $\rho_{i}$ :

$$
\left(2 B \rho_{i}-1\right) e^{\frac{1}{B \rho_{i}}}-\left(2 B \rho_{i}+1\right)=0
$$

This does not have a solution, but the left side of the equation is always negative and only approaches zero asymptotically for $\rho_{i} \rightarrow \infty$.

\footnotetext{
${ }^{4}$ This is the point where working with the SFM in $2 \mathrm{~d}$ would take a different direction, since it would be rather $\sqrt{\rho}=1 / d_{0}$ - if a $d_{0}$ could be defined at all - and the following math might yield different results.
} 
We note the first definite result of this work:

Result 1: Neither the circular specification nor the elliptical specification II of the Social Force Model as originally defined produce an inflection point in the speed density relation for homogeneous steady-state one-dimensional movement.

\section{The Social Force Model for Steady-States in Single-File Movement with only Nearest Neighbor Interaction}

This result naturally raises the question if in the face of empirical data it is time to sing the "Requiem for the Social Force Model" or if it can be resurrected. The fact that Eq. 32 can be solved at least asymptotically is sufficient motivation to search for model modification that lead to a solution at finite values for $\rho_{i}$. Indeed different solutions can be found, probably the simplest is described in this section.

We reconsider Eq. 23 and investigate a variant of the model where not all pedestrians, but only the nearest neighbors - the one in front and the one at the rear - exert a force on pedestrian $\alpha$. This leads to

$$
\dot{x}_{\alpha}=v_{0}-(1-\lambda) \tau A e^{-\frac{1}{B \rho}}
$$

We note that Eq. 33 is different from Eq. 1 only through parametrization. The functional form is identical. The parameter equivalents are

$$
\begin{aligned}
v_{0} & =v_{f} \\
\frac{1}{B} & =\gamma \\
\frac{(1-\lambda) \tau A}{v_{0}} & =e^{\frac{\gamma}{\rho_{m}}}
\end{aligned}
$$

We note as second result of this work:

Result 2: If the circular specification or the elliptical
specification II of the Social Force Model are mod-
ified such that only nearest neighbors exert a force
onto each other, then the speed density relation for ho-
mogeneous steady-state one-dimensional movement
is given by the Kladek formula. Therefore the Kladek
formula is a macroscopic description of a modified
Social Force Model.

Since the Kladek formula has the inflection point as desired, so does the SFM with only nearest-neighbor interactions. This matches in approach and result with [33] where 



Figure 7 Speed-density (left) and flow-density (right) relations if a different number of neighbors $(1,2,4$, $8, \infty)$ is considered to both sides for the computation of forces in the Social Force Model. The plots are based on the dimensionless variants of equations and it is $a=0.2$.

also a nearest-neighbor model produced an inflection point and positive curvature at high densities in the speed-density relation.

Thus if one trusts the Kladek formula on a macroscopic level it is at least beneficial if not required for any (force-based) model of pedestrian dynamics if it reduces to the circular specification of the Social Force Model when uni-directional single-file steadystate movement with a homogeneous population is described and simulated. An example for which this is not the case is elliptical specification I of the Social Force Model [32, $34,34]$ which frequently is termed the Social Force Model. For elliptical specification I it is not possible to give a closed analytical form of the speed-density relation. Furthermore the resulting implicit speed-density equation for single-file uni-directional steady state homogeneous movement raises the suspicion that - at least for certain parameter values - speeds above the desired speed may occur for finite densities. In any case elliptical specification I is not covered by this work and would need a separate treatment.

If in the Social Force Model next and next-to-next neighbors exert a force onto each other the resulting (dimensionless) speed density relation modifies to

$$
f(x)=1-\frac{e^{-\frac{a}{x}}+e^{-2 \frac{a}{x}}}{e^{-a}+e^{-2 a}}
$$

and the condition for the inflection point modifies to

$$
e^{-\frac{a}{x_{i}}}=-\frac{2 x_{i}-a}{4 x_{i}-4 a}
$$

which for example for a parameter value $a=1$ is solved by $x_{i} \approx 0.65$ and which has solutions $x_{i}<1$ as long as $0<a<\approx 1.53$. Analytical treatment becomes more difficult, but one can easily plot speed density relations that result from taking an arbitrary number of neighbors (in both directions) into account. Fig. 7 shows some of them.

Considering $N$ nearest neighbors for the calculation of the forces implies that in fact there are $N$ variants of the original Social Force Model (specifications) - one may call these briefly "SFMn". And all of them - except for $N=1$ - motivate a different modification and extension of the Kladek formula. The first thought might be that this offers a lot 
of freedom for calibration and that with sufficient empirical data available one would be able to pick the "right" value of $N$ for a realistic variant of the Kladek formula as well as a realistic variant of the Social Force Model. However two arguments speak against this: first - at least with a second thought - it is intuitively clear that SFM parameters obtained from calibration in a really crowded scene, maybe resulting in a relatively high value for $N$ might not necessarily work well for simulations when just two pedestrians meet and evade. Second, even if various calibration processes would give a consolidate result for $N$ - say $N=42$ - the question would be "Why 42?". There is no intuitively comprehensible argument that could support the result of the calibration; no matter what the resulting number for $N$ was. So, while considering only a limited number of pedestrians for the computation of forces can be seen as a step forward, it is nevertheless desirable to find a different approach.

\section{The Social Force Model for Steady-States in Single-File Movement with Neighborhood Degree dependent Force Suppression}

As a first step we write Eq. 33 seemingly unnecessarily complicated as

$$
\dot{x}_{\alpha}=v_{0}-(1-\lambda) \tau A \frac{1}{e^{\frac{1}{B \rho}}-0}
$$

In this form we note that the only difference between Eq. 28 and 39 - so between the original formulation where infinitely many pedestrians exert a force on $\alpha$ and the nearest neighbor variant - is only that in the first case there is a " 1 " and in the second case a " 0 " in the denominator.

Having written the equations in this way the natural question is "What if instead of the zero or one in Eq. 28 and 39 we write there some $0<k<1$ ?":

$$
\dot{x}_{k \alpha}=v_{0}-(1-\lambda) \tau A \frac{1}{e^{\frac{1}{B \rho}}-k}
$$

respectively in dimensionless form:

$$
f(x)_{k}(\rho)=1-\frac{e^{a}-k}{e^{\frac{a}{x}}-k}
$$

This modifies the second derivative:

$$
\begin{aligned}
v_{k}(\rho) & =v_{0}-(1-\lambda) \tau A \frac{1}{e^{\frac{1}{B \rho}}-k} \\
\frac{\partial v_{k}(\rho)}{\partial \rho} & =-(1-\lambda) \tau A \frac{e^{\frac{1}{B \rho}}}{B \rho^{2}\left(e^{\frac{1}{B \rho}}-k\right)^{2}} \\
\frac{\partial^{2} v_{k}(\rho)}{\partial \rho^{2}} & =(1-\lambda) \tau A e^{\frac{1}{B \rho}} \frac{(2 B \rho-1) e^{\frac{1}{B \rho}}-k(2 B \rho+1)}{B^{2} \rho^{4}\left(e^{\frac{1}{B \rho}}-k\right)^{3}}
\end{aligned}
$$




\begin{tabular}{ll|ll|ll}
\hline$k$ & $B \rho_{i}$ & $k$ & $B \rho_{i}$ & $k$ & $B \rho_{i}$ \\
\hline 0.0 & 0.500 & 0.90 & 0.981 & 0.99 & 2.049 \\
0.1 & 0.515 & 0.91 & 1.013 & 0.999 & 4.379 \\
0.2 & 0.531 & 0.92 & 1.051 & 0.9999 & 9.416 \\
0.3 & 0.551 & 0.93 & 1.096 & 0.99999 & 20.28 \\
0.4 & 0.576 & 0.94 & 1.151 & 0.999999 & 43.68 \\
0.5 & 0.606 & 0.95 & 1.219 & 0.9999999 & 94.10 \\
0.6 & 0.646 & 0.96 & 1.309 & 0.99999999 & 202.7 \\
0.7 & 0.703 & 0.97 & 1.435 & 0.999999999 & 436.8 \\
0.8 & 0.793 & 0.98 & 1.635 & 0.9999999999 & 941.0 \\
\hline
\end{tabular}

Table 1 Numerical solutions for the value $\rho_{i}$ of the inflection point with regard to various values for parameter $k$. Values computed with [35]
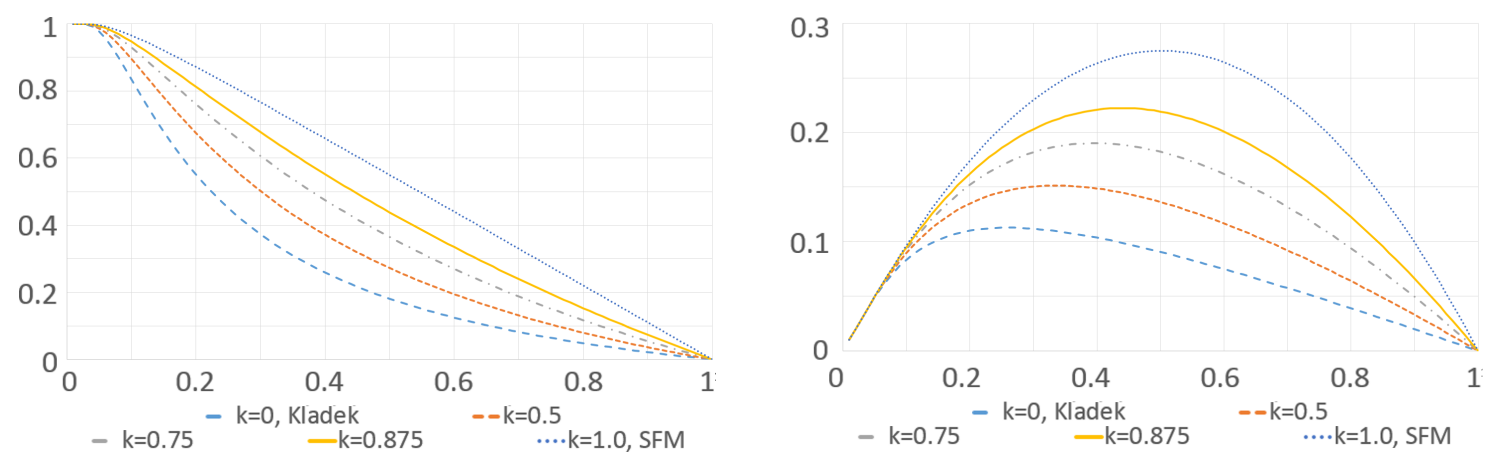

Figure 8 Speed-density (left) and flow-density (right) relations of the $k$-extended Social Force Model for various values of parameter $k$. The plots are based on the dimensionless variants of equations and it is $a=0.2$.

For $0 \leq k<1$ the new equation to solve is

$$
\left(2 B \rho_{i}-1\right) e^{\frac{1}{B \rho_{i}}}-k\left(2 B \rho_{i}+1\right)=0
$$

and it has a solution. For $k=0$ it is obviously $\rho_{i}=1 /(2 B)$ (in agreement with the discussion of the Kladek formula). For other values of $k$ the solution needs to be computed numerically. Tab. 1 gives some values for $B \rho_{i}$.

The introduction of parameter $k$ produces the desired inflection point in the speeddensity diagram. See Fig. 8.

Next we try to find an interpretation for that introduction which so far was of purely mathematical-technical nature. We write Eq. 42 in a slightly different manner

$$
v_{k}(\rho)=v_{0}-(1-\lambda) \tau A \frac{1}{k} \frac{1}{\frac{e^{\frac{1}{B \rho}}}{k}-1}
$$


and undo the summation of the geometric series with $k$ "on the back" of the exponential function

$$
\begin{aligned}
v_{k}(\rho) & =v_{0}-(1-\lambda) \tau A \frac{1}{k} \sum_{n=1}^{\infty} k^{n} e^{-\frac{n}{B \rho}} \\
& =v_{0}-(1-\lambda) \tau A \sum_{n=1}^{\infty} k^{n-1} e^{-\frac{n}{B \rho}} \\
& =v_{0}-(1-\lambda) \tau A \sum_{n=1}^{\infty} k^{n-1} e^{-n \frac{d_{0}}{B}}
\end{aligned}
$$

In the process of simplifying the SFM with Eq. 24 it was assumed that pedestrians are equally spaced $\left(n d_{0}=d_{\alpha \beta}\right)$. Now we drop this simplification, allowing varying distances, and define a new, extended model. This new model reduces to the macro relations of Eq. 42 and 48 if the simplifying assumptions of equilibrium and equal spacing are made. The mathematical transformation in this step is trivial $\left(n d_{0}\right.$ is replaced by $\left.d_{\alpha \beta}\right)$, the main change is that the sum has to run over pedestrians $\beta$ not over numbers $n$. Nevertheless a $n$ remains in the sum, but now it is a $n$ associated with a particular pedestrian $\beta$ and therefore denoted $n_{\beta}$. It is important to keep in mind that the value of $n_{\beta}$ cannot be determined by looking at pedestrians $\alpha$ and $\beta$ alone, but that one needs to know the positions of all other pedestrians as well.

$$
\ddot{x}=\frac{v_{0}-v}{\tau}-(1-\lambda) A \sum_{\beta}^{\infty} k^{n_{\beta}-1} e^{-\frac{d_{\alpha \beta}}{B}}
$$

This is easy to interpret: the next neighbors of $\alpha$ exert a force on $\alpha$ unmodified compared to the original SFM. The second next neighbors exert a force which is suppressed by a factor $k$ (always compared to the original model without parameter $k$ ). The force from the next to next to next nearest neighbors is suppressed by a factor of $k^{2}$ and so on. This implies for example that if we take out each second pedestrian from a simulation and the next to next nearest neighbor becomes the next nearest neighbor the force from this new nearest neighbor is larger than before when s/he was just the next to next nearest neighbor although the distance to $\alpha$ is the same as before.

One can argue that such a modification makes intuitively sense. If two pedestrians approach "me" independently and the first remote pedestrian overtakes and becomes the closest one "my" awareness is shifted from the former to the new nearest neighbor and that would have an impact on my velocity changes, i.e. acceleration, i.e. forces. This intuitive comprehensibility of the model extension adds to the pleasure of having gained the desired inflection point. Fig. 9 summarizes this visually. 

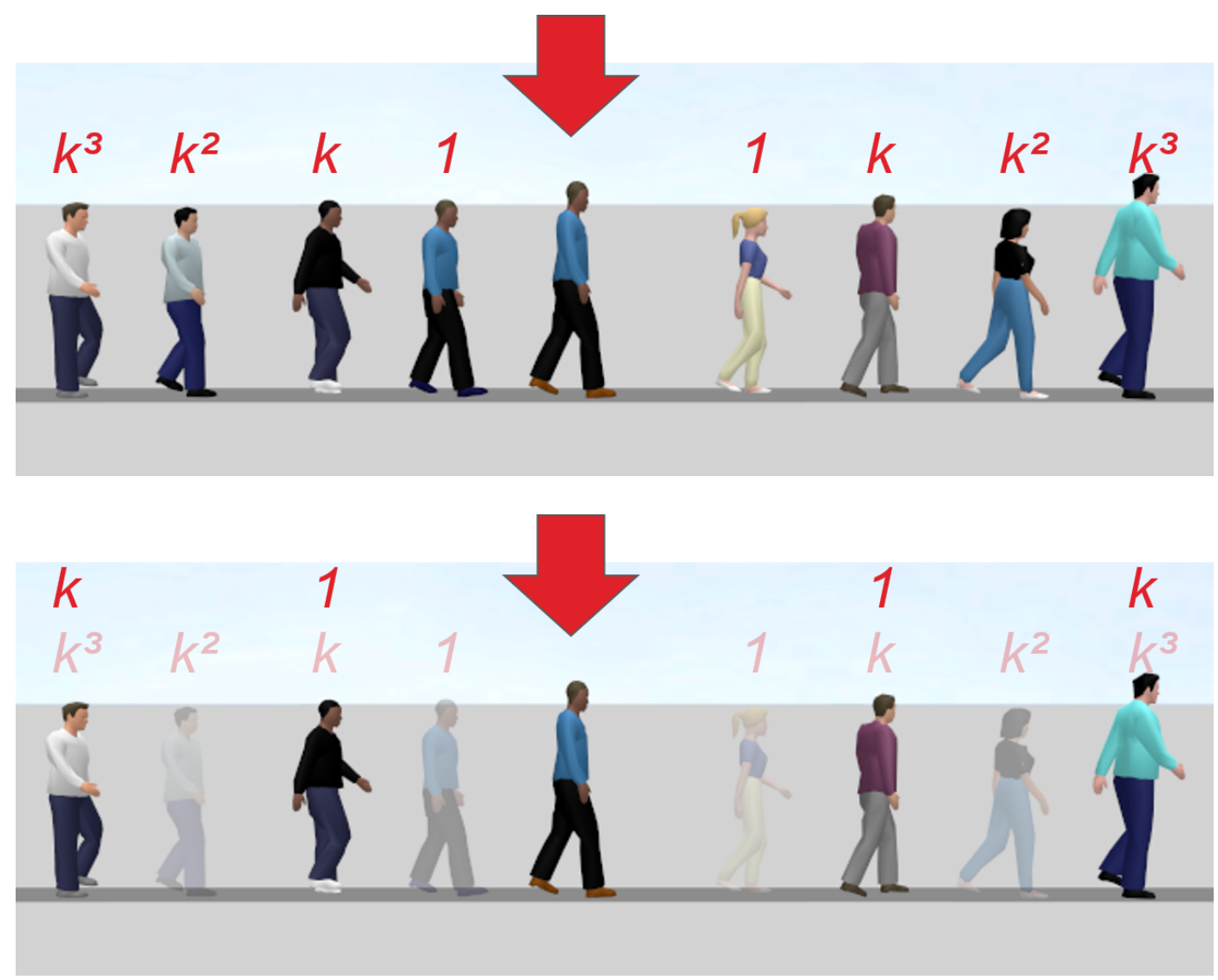

Figure 9 From the upper to the lower picture density is reduced by half. If we ask what might be the force(s) on the person marked with an arrow, we may intuitively argue that the person who is the second next (brown hair, violet shirt, gray trousers) in the upper picture has a higher impact on the arrow-marked guy at the moment the blonde-blue-white lady disappears and he becomes the next nearest neighbor, although the distance between the two is exactly the same as before. This is exactly what the $\mathrm{k}$ extension of the SFM does in this case: the $k<1$ factor on the force disappears and the force value increases.

Result 3: Suppressing the force from each pedestrian
in the sequence ordered by distance from the pedes-
trian for whom forces are calculated with an addi-
tional factor $0<k<1$ produces an inflection point
in the resulting speed-density relation and can as a
model extension be motivated intuitively.

Further favorable properties of this model extension are that it can be combined with arbitrary specifications of the SFM or in general force-based models as for example [36-39] and that the new parameter $k$ re-opens the possibility for calibration even if one requires that the maximum of the flow be at same density as the inflection point in the speeddensity relation.

We would like to emphasize that the introduction of parameter $k$ as extension of the Social Force Model brings a major conceptual change. Without it forces of various pedes- 
trians superpose, i.e. the force of one pedestrian $\beta_{1}$ on some pedestrian $\alpha$ does not depend on if there are other pedestrians $\beta_{i}$ and if they are there where they are located and with which velocity they move. This changes with parameter $k$. Now one has to know the local distribution of all pedestrians before one can compute the force of one pedestrian on another one. Forces do not superpose anymore. Instead the extended model - let us briefly call it "SFMk" - is rather described by Sherif's famous description of social systems: "the properties of any part are determined by its membership in the total functional system." [40]. In this sense the SFMk structurally bears some similarities to another extension of the Social Force Model - namely the dynamic potential [41-44] - where the desired direction of pedestrians is computed such that they walk into the direction of earliest expected arrival, for this considering the distribution and movement state of all pedestrians in a holistic way. A difference, however, between SFMk and the dynamic potential is that in SFMk it is exactly known which pedestrian $\beta_{i}$ contributes what to the effect on the movement of pedestrian $\alpha$. This is not the case for the dynamic potential. We would therefore call the dynamic potential a mesoscopic or mean-field or holistic modeling element while the SFMk is non-superposing but non-holistic and entirely microscopic.

\section{Comparing the Model Modifications SFMn and SFMk}

Concerning the relation and difference of SFMn and SFMk models we find for once that for a given value of $a$ for each value of $N$ there is a narrow range of $k$ values with which the speed-density relations match closely, but never exactly. It is always the case that for small densities the SFMn model and for high densities the SFMk model gives higher speeds; see Fig. 10. Still the differences are marginal such that for practical applications corresponding models should always be exchangeable as far as only the speed-density relation is concerned. Tab. 2 gives pairs of corresponding values for $N$ and $k$ for three different values of parameter $a$. Second, there are values for $k$ for which - again for a given value of parameter $a$ - the speed-density relation of the SFMk model lies (almost) entirely between two "neighboring" SFMn models $(N$ and $N+1)$, thereby giving the impression that for small densities the SFMk model gives rather speeds like the SFMn model with smaller $N$ and for higher densities like the one with $N+1$. See Fig. 11 .

\section{Comparison of SFMk to Empirical Data}

Before we do the comparison we would like to state its limitations.

There is first the obvious difference between the theoretical system which was assumed to be infinitely large and the necessarily finite size laboratory system which produced empirical data. Arguing that the closed-loop laboratory system can straightforwardly be interpreted as a system with periodic boundary conditions would imply for a simulation with the Social Force Model that a person exerts some force on itself. He/she would do so infinitely often from the front with a decelerating effect and infinitely often from behind with an accelerating effect. For a single pedestrian alone in the loop of length $L$ this would 

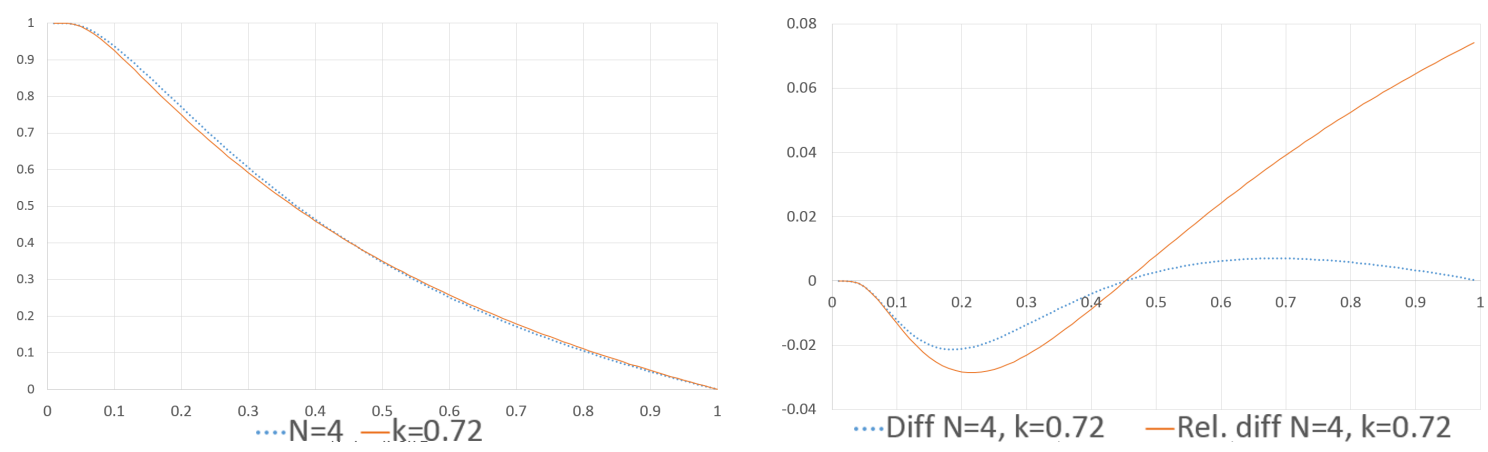

Figure 10 Left: Speed-density relation for SFMn with $N=4$ and SFMk with $k=0.72$. Right: Difference and relative difference between both. The plots are based on the dimensionless variants of equations and it is $a=0.2$.

\begin{tabular}{l|l|l|l}
\hline$N$ & $k$, with $a=0.02$ & $k$, with $a=0.2$ & $k$, with $a=2.0$ \\
\hline 1 & 0.00 & 0.00 & 0.00 \\
2 & 0.35 & 0.42 & 0.90 \\
3 & 0.53 & 0.63 & 1.00 \\
4 & 0.64 & 0.72 & 1.00 \\
6 & 0.75 & 0.84 & 1.00 \\
8 & 0.81 & 0.90 & 1.00 \\
10 & 0.85 & 0.94 & 1.00 \\
12 & 0.87 & 0.96 & 1.00 \\
16 & 0.91 & 0.98 & 1.00 \\
20 & 0.93 & 0.99 & 1.00 \\
$\infty$ & 1.00 & 1.00 & 1.00
\end{tabular}

Table 2 Approximate corresponding values for $N$ and $k$ for the SFMn and SFMk models with $a=0.02$, $a=0.2$, and $a=2.0$.


Figure 11 Left: Speed-density relation for two SFMn models $(\mathrm{N}=2$ and $\mathrm{N}=3)$ and one SFMk model $(k=0.6)$. Right: Differences between the SFMk and the SFMn models. The plots are based on the dimensionless variants of equations and it is $a=0.2$. 
imply that by self-interaction the walking speed would be

$$
\frac{v}{v_{0}}=1-\frac{(1-\lambda) \tau A}{e^{\frac{L}{B}}-1}
$$

with for example $\lambda=0.1, \tau=0.5 \mathrm{~s}, A=10 \mathrm{~m} / \mathrm{s}^{2}, B=3 \mathrm{~m}$, and $L=17.3 \mathrm{~m}$ the pedestrian would slow down by $1.4 \%$. For typical parameters and the size of the experiment the effect is small, but we need to keep in the back of the mind that the assumption that the infinite and the closed system correspond implies already that we also assume that interactions between pedestrians in fact are of limited range. If they were not, if another pedestrian 10 to $20 \mathrm{~m}$ away and with a couple of people in between would have a significant effect, in reality it would make a difference if this other person is the same or a different person than the person whom we are concerned about (for whom movement is calculated) and then it would not be justified to compare results from finite size and closed, and open and infinite systems. However, since the results for the short and the long loop as shown on the right side of Fig. 3 match very closely, we may assume that already with the short loop the experimenters implemented a system size which is large compared to relevant pedestrian interaction distance, meaning that one can expect that increasing the size ever further would not yield any different results, justifying in consequence our assumption.

Second, we will assume that the average of data, i.e. the central line through the clouds of data points, corresponds to the stationary state of the continuous description. This is of course not guaranteed, especially since it is not guaranteed that a stable stationary state exists at all. The justification for this assumption would be made ex post: if simulating microscopically with parameter values that have been gained from matching the continuous stationary description to the scattered empirical data produces realistic results - better: more realistic results than parameter values used before - then the approach was justified.

\subsection{Comparison to Empirical Data of Pedestrians}

The theory so far was straight forward and one might expect that calibration of the resulting equations at empirical data should be equally straight forward. However, there are four issues: first, the theory assumes nonchalant that the average free speed and the maximum density are known. Yet measuring a meaningful average speed near maximum density is difficult or even impossible since one is faced with stop-and-go waves in pedestrian [5,45] as well as vehicular traffic.

Second, also for all other densities the empirical data exhibits a wide scattering. One might choose an averaging method that produces from the scattered empirical data unique speed values for a number of density values, however since there are various such methods, the choice already bears some arbitrariness. Or one can fit the functions "cloudbased" by eye-sight comparison. Lacking the original empirical data, in this section we proceed with the second method. With this approach numerous functions can appear equally well fitting and Fig. 12 shows that these functions may have quite different parameters. 


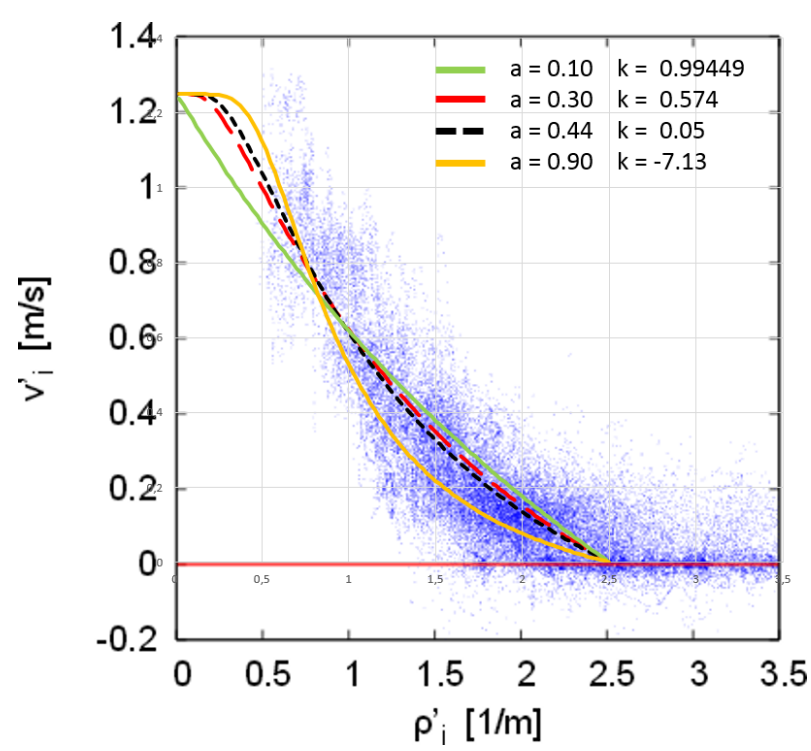

Figure 12 Four functions with different parameters displayed on the background of the left figure of Fig. 4. Free speed and maximum density were not calibrated, but were fixed a priori to $v_{0}=$ $1.25 \mathrm{~m} / \mathrm{s}$ and $\rho_{\max }=2.51 / \mathrm{m}$.

Third, there is few to none data available that has been collected and averaged in the momentary, space-averaged way. None of the data referenced so far in this work fit this requirement exactly. The speed data in Fig. 2 and 3 has been obtained by measuring the time it took pedestrians to walk a fixed distance. In this way very low speeds are systematically underrepresented compared to a measurement where speed is measured by dividing the distance pedestrians move in a fixed time and speed zero can never occur in contrast to Kladek's formula. The speed data in the left diagram of Fig. 4 has been obtained in the latter way, but density data was not measured in the usual space-averaging way. The right diagram in the same figure shows space-averaged data, but not stated explicitly if speeds were measured with fixed $\Delta t$ or fixed $\Delta x$. Definitely a problem is that a measurement was triggered each time a pedestrian crossed position $x=0$ which means that if density was so high that no one could move no measurement was triggered. This implies that - although data is space-averaged - small speeds are systematically suppressed compared to when measurement is triggered externally. However, since overtaking is not possible in these scenarios one can hope that the impact of the averaging method is rather small. Still one has to bear the stated limitations in mind when interpreting the results of this section.

In Fig. 12 one of the four functions even has a negative value for parameter $k$. This does not make sense with regard to the Social Force Model since then each second pedestrian would have an attractive and each second pedestrian a repelling effect. However, mathematically it is possible to apply $k$ s with negative value.

\subsection{Comparison to Empirical Data of Pedestrians, Cyclists, and Cars}

Recently a comparison has been done between the speed-density diagrams of one-dimensional, uni-directional car, bike, and pedestrian traffic [46,47]. For this the already 

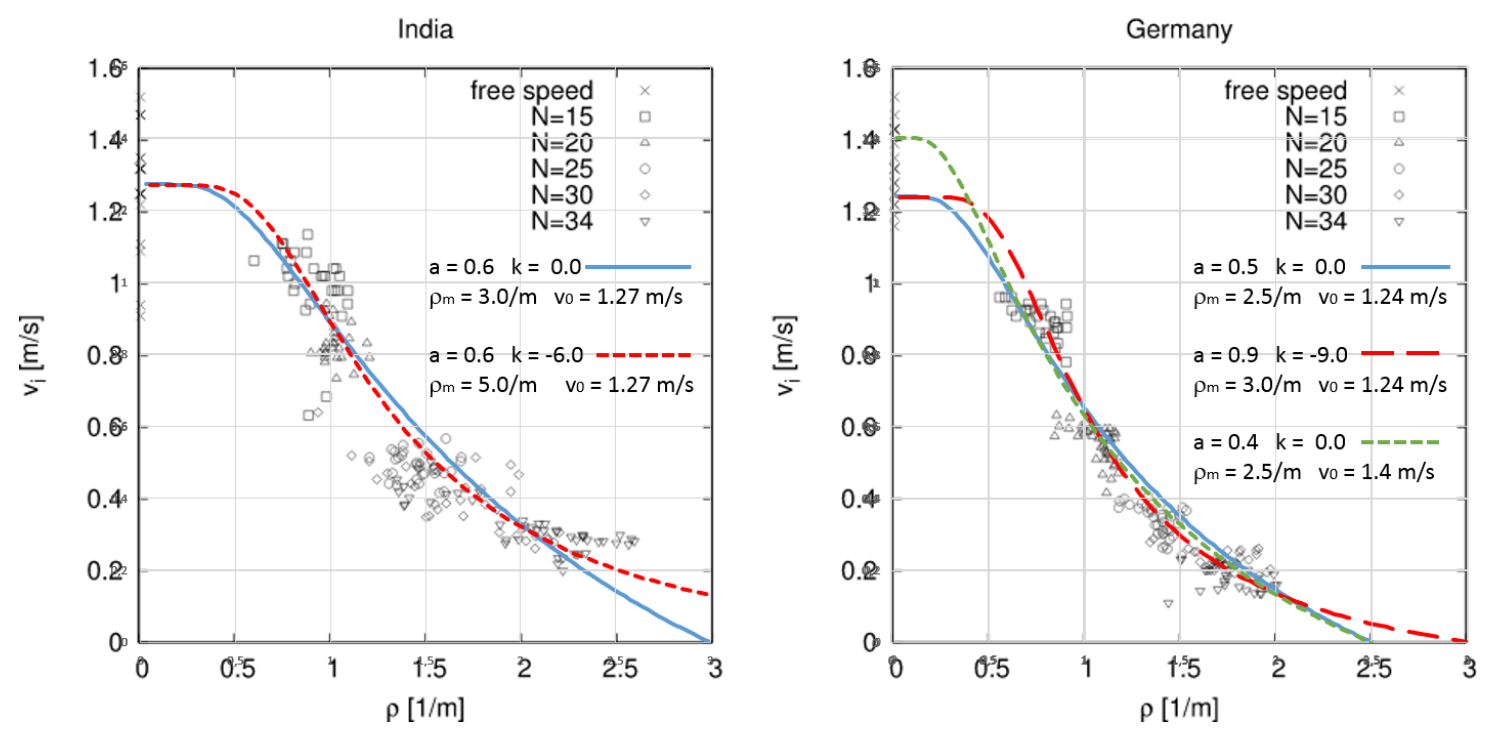

Figure 13 Various functions overlaid with Fig. 2

referenced data for pedestrians has been used, new data for cycling dynamics has been gathered and existing data from an experiment on vehicular dynamics [48, 49] has been added. According to the description of measurement and (re-)evaluation here speed has been measured in exact accordance with the definition for quasi-instantaneous, spaceaveraged measurement. This makes the theory discussed in this contribution applicable to all three modes of traffic and it is interesting to see if the parameter values of $a$ and $k$ vary over the modes.

From Fig. 14 one may suspect that $k_{c a r}>k_{\text {bike }}>k_{\text {ped }}$ and conclude from that that car drivers look more ahead (react more to the next to next and further leading vehicle) than cyclists and those more than pedestrians do. One can further conclude that the Kladek formula where in effect $k=0$ fits better to pedestrian dynamics although it was introduced for vehicular (urban) traffic while the Social Force Model where in effect $k=1$ describes rather vehicular dynamics although it was suggested as a model of pedestrian dynamics. However with all limitations discussed above all conclusions must be considered to be very preliminary.

\section{Summary - Discussion - Conclusions - Outlook}

In this contribution we have computed the continuous description of the Social Force Model of pedestrian dynamics for single-file uni-directional movement. We found that the speed-density curve does not have an inflection point, but that with the introduction of one more parameter to the Social Force Model one can recover an inflection point and at the same time transfer the macroscopic description of the Social Force Model continuously to the Kladek formula.

In the model extension - called SFMk - forces from other pedestrians are suppressed by neighborhood degree in addition to the usual decay of forces in the SFM that goes 


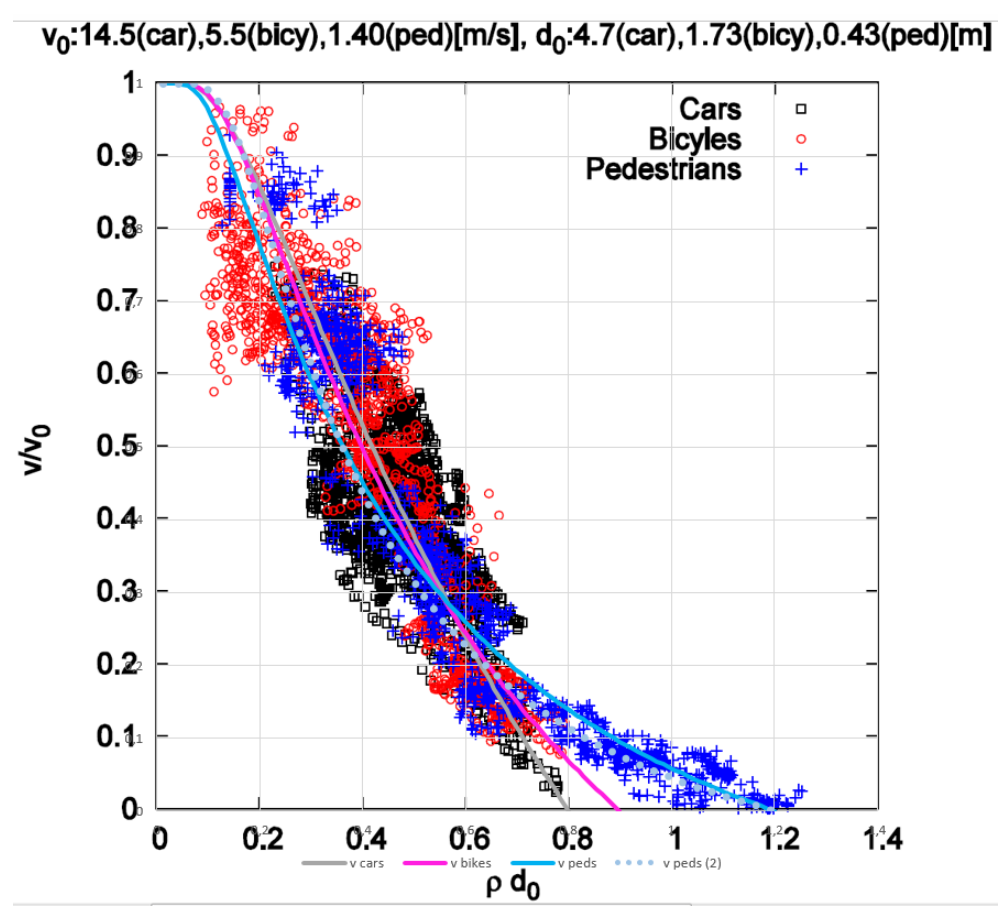

Figure 14 Figure 7b from [46] overlaid with SFMk functions. Note that in the diagram "density=1" marks full occupancy (212.8 cars/km corresponding to $4.7 \mathrm{~m}$ per car; $578 \mathrm{bikes} / \mathrm{km}$, corresponding to $1.73 \mathrm{~m}$ per bike; 2325.6 pedestrians per $\mathrm{km}$, corresponding to $0.43 \mathrm{~m}$ per pedestrian) and "speed=1" marks the maximum speed (cars: $14.5 \mathrm{~m} / \mathrm{s}$; bikes: $5.5 \mathrm{~m} / \mathrm{s}$ and pedestrians: 1.4 $\mathrm{m} / \mathrm{s}$ ). Thus different to our approach in the paper density $=1$ marks the theoretically maximum density not the actual one. Please note the comments in [46] why densities larger 1 have been measured for pedestrians. The gray curve is our approximation to car data. It has $a=0.5$, $k=0.7$, and $x_{\max }=0.8$. The magenta curve is our approximation to bike data. It has $a=0.5$, $k=0.2$, and $x_{\max }=0.9$. The cyan curve is our approximation to pedestrian data with the restriction that it has to be $k \geq 0$. It has $a=0.3, k=0.0$, and $x_{\text {max }}=1.2$. The dotted light blue is our approximation to pedestrian data without restriction for $k$. It has $a=0.5, k=-2.0$, and $x_{\text {max }}=1.2$. Maximum speed is 1 for all curves.

exponentially with distance. We also discussed a different model modification - called SFMn - where forces are computed unmodified, but only the $N$ nearest neighbors are taken into account, and discovered that with appropriate parametrization SFMk and SFMn exhibit very similar speed-density relations.

A comparison with empirical data of pedestrian dynamics suggests that both model modifications yield more realistic results. A further look on comparable empirical data for cyclist and car dynamics suggests that car drivers might consider next-to-next and further vehicles more for their own movement decisions, while pedestrians concentrate most on the person(s) immediately neighbored.

These observations suggest the conclusion that it would be at least beneficial, if not absolutely required, to use the SFMk model extension in pedestrian planning simulation projects. However, before this is brought into practice two issues have to be considered or resolved:

First, a SFMk-type model for each pedestrian in each simulation time step would re- 
quire a sorting of surrounding pedestrians by their distance or - see below - other properties. This could prove to be computationally costly and slow simulations down. The SFMn model variant could be an appropriate compromise. In PTV Viswalk for example parameter ReactToN in the walking behaviors specifies to how many pedestrians some pedestrian will react. With a default of ReactToN $=8$ this is a SFM $n=4$ model [50]. The SFMn contrary to SFMk means that simulation time is reduced compared to the original SFM, since much fewer inter-pedestrian forces have to be computed.

Second - and this is the actual outlook on future activities - the SFMk proposed here is a one-dimensional model. For application pedestrians need to be simulated in two space dimensions. Unfortunately the extension of the SFMk to two dimensions is not straight forward, since in $2 \mathrm{~d}$ the obvious ordering is lost and it is not trivial to decide which power of $k$ has to be assigned to which pedestrian. One could of course simply order pedestrians by distance (variant A). Or to take into account that in the $1 \mathrm{~d}$ case for each power of $k$ there is a pedestrian in front and one behind one could partition the area around a pedestrian fan-like into slices and count pedestrians separately in each slice (variant B). It is not clear, however, which number of slices would make up for a good choice. Therefore it appears to us most promising to do a Voronoi tessellation based on the positions of pedestrians and assign $k^{0}$ to all pedestrians in nearest Voronoi cells, $k^{1}$ to all pedestrians in next-to-nearest Voronoi cells etc. (variant C). Variants A, B, and C share in common that they all depend on spatial separation (i.e. distance) as ordering property. It is not obvious, however, that this will yield the best results. One could also think of a time based ordering (time to collision, post encroachment time) or other safety surrogate measures like deceleration to safety time [51]. A safety surrogate-based ordering would require a hierarchical method: first identify those pedestrians with whom there are potential future collision issues and order them accordingly, then order the remaining pedestrians according to a different property.

From a theoretical perspective there is - or at least might be - further potential for improvements under the heading of an "inflection point"; this time not in the speed-density, but the flow-density or flow-occupancy function, since the model modifications proposed here do NOT induce an inflection point in the flow-density function. However, there are two reasons to assume that one such ought to exist. The first is that it was directly observed, at least with certain measurement methods [52], the second reasoning is indirect: it could recently be shown by evaluation of empirical data that in pedestrian dynamics there are fairly stable stop-and-go-waves [45]; and for the LWR model [53] it is known that stable start waves only exist if and where the curvature of the flow-occupancy function is positive [54]. Thus for density waves ("humps") that have a stable stop and a stable start front, it requires a fundamental diagram which has parts with negative and parts with positive curvature above capacity, unless a different mechanism which is not captured by the LWR model is found that can explain stability. Usually instabilities i.e. spontaneously or from small perturbations evolving density humps - in transportation systems are explained by and modeled with retarded reactions of the units [55]. Yet since there is no retarded reaction in the SFM, at least not explicitly, extending the model such that the resulting fundamental diagram has an inflection point above capacity can be an alternative to adding finite reaction times. One can even think of that possibly introducing 
retarded reaction is exactly what may produce the inflection point. For example the introduction of retarded reaction particularly for acceleration from standstill ("slow-to-start rule") produces an inflection point in the fundamental diagram of the $T^{2}$ model of car traffic [56,57].

\section{References}

[1] Seyfried, A., Steffen, B., Klingsch, W., Boltes, M.: The fundamental diagram of pedestrian movement revisited. Journal of Statistical Mechanics: Theory and Experiment 2005(10), P10002 (2005). doi:10.1088/1742-5468/2005/10/P10002

[2] Chattaraj, U., Seyfried, A., Chakroborty, P.: Comparison of pedestrian fundamental diagram across cultures. Advances in Complex Systems 12(03), 393-405 (2009). doi:10.1142/s0219525909002209

[3] Seyfried, A., Portz, A., Schadschneider, A.: Phase coexistence in congested states of pedestrian dynamics. In: Bandini, S., Manzoni, S., Umeo, H., Vizzari, G. (eds.) Cellular Automata, Lecture Notes in Computer Science, vol. 6350, pp. 496-505. Springer Berlin Heidelberg (2010)

[4] Seyfried, A., Boltes, M., Kähler, J., Klingsch, W., Portz, A., Rupprecht, T., Schadschneider, A., Steffen, B., Winkens, A.: Enhanced empirical data for the fundamental diagram and the flow through bottlenecks. In: Pedestrian and Evacuation Dynamics 2008, pp. 145-156. Springer (2010)

[5] Portz, A., Seyfried, A.: Analyzing stop-and-go waves by experiment and modeling. In: Peacock, R.D., Kuligowski, E.D., Averill, J.D. (eds.) Pedestrian and Evacuation Dynamics, pp. 577-586. Springer US (2011)

[6] Weidmann, U.: Transporttechnik der Fussgänger: Transporttechnische Eigenschaften des Fussgängerverkehrs (Literaturauswertung). ETH, IVT (1993)

[7] Buchmueller, S., Weidmann, U.: Parameters of pedestrians, pedestrian traffic and walking facilities. Institute for Transport Planning and Systems (IVT), Chair of Transport Systems, ETH Zurich (2006)

[8] Kladek, H.: Über die Geschwindigkeitscharakteristik auf Stadtstraßenabschnitten. $\mathrm{Ph} . D$. thesis, Hochschule für Verkehrswesen „Friedrich List” Dresden (1966)

[9] Newell, G.: Nonlinear effects in the dynamics of car following. Operations research 9(2), 209-229 (1961). doi:10.1287/opre.9.2.209

[10] Lohse, D., Schnabel, W.: Grundlagen der Straßenverkehrstechnik und der Verkehrsplanung: Band 1-Verkehrstechnik, 3 edn. Beuth Verlag (2011) 
[11] Wu, N.: Verkehr auf Schnellstraßen im Fundamentaldiagramm. Straßenverkehrstechnik 8, 378-388 (2000)

[12] Kühne, R.: Das Fundamentaldiagramm-Grundlagen und Anwendungen. FGSV Merkblatt (Entwurf) p. 59 (2004)

[13] Daamen, W.: Modelling passenger flows in public transport facilities. Ph.D. thesis, Delft University of Technology (2004)

[14] Bellomo, N., Dogbe, C.: On the modelling crowd dynamics from scaling to hyperbolic macroscopic models. Mathematical Models and Methods in Applied Sciences 18(supp01), 1317-1345 (2008). doi:10.1142/s 0218202508003054

[15] Lämmel, G., Rieser, M., Nagel, K.: Bottlenecks and congestion in evacuation scenarios: A microscopic evacuation simulation for large-scale disasters. In: Proc. of 7th Int. Conf. on Autonomous Agents and Multiagent Systems (AAMAS 2008), Estoril, Portugal. Citeseer (2008)

[16] Lämmel, G., Klüpfel, H., Nagel, K.: The MATSim network flow model for traffic simulation adapted to large-scale emergency egress and an application to the evacuation of the Indonesian city of Padang in case of a tsunami warning, chap. 11, pp. 245-265. Emerald Insight (2009). doi:10.1108/9781848557512-011

[17] Venuti, F., Bruno, L.: Crowd-structure interaction in lively footbridges under synchronous lateral excitation: A literature review. Physics of Life Reviews 6(3), 176206 (2009). doi:10.1016/j.plrev.2009.07.001

[18] Beltaief, O., El Hadouaj, S., Ghedira, K.: Multi-agent simulation model of pedestrians crowd based on psychological theories. In: Logistics (LOGISTIQUA), 2011 4th International Conference on, pp. 150-156. IEEE (2011). doi:10.1109/LOGISTIQUA.2011.5939418

[19] Galiza, R., Ferreira, L., Charles, P.: Estimating the effects of older people in pedestrian flow: A micro-simulation approach. In: Transport Research Board TRB 90th Annual Meeting, pp. 1-13. Transportation Research Board (TRB) (2011)

[20] Papadrakakis, M., Fragiadakis, M., Plevris, V.: Footbridge lateral vibrations induced by synchronised pedestrians: An overview on modelling strategies. In: 3rd ECCOMAS Thematic Conference on Computational Methods in Structural Dynamics and Earthquake Engineering (2011)

[21] Bruno, L., Tosin, A., Tricerri, P., Venuti, F.: Non-local first-order modelling of crowd dynamics: A multidimensional framework with applications. Applied Mathematical Modelling 35(1), 426-445 (2011). doi:10.1016/j.apm.2010.07.007

[22] Bruckner, M., Tauböck, S., Popper, N., Emrich, Š.., Rozsenich, B., Alkilani, S.: A combined cellular automata and devs simulation. In: MathMod 2012 (2012) 
[23] Huth, F., Bärwolff, G., Schwandt, H.: Fundamental diagrams and multiple pedestrian streams. Preprint 2012/17 p. eprint (2012)

[24] Lüßmann, J., Sánta, C.: Network-wide evaluation of cooperative traffic systems using microscopic traffic flow simulation. Simulation Notes Europe p. 89 (2012). doi:10.11128/sne.22.tn.10129

[25] Zhang, J.: Pedestrian fundamental diagrams: Comparative analysis of experiments in different geometries. Ph.D. thesis, Forschungszentrum Jülich (2012)

[26] Nikolić, M., Bierlaire, M., Farooq, B.: Probabilistic speed-density relationship for pedestrians based on data driven space and time representation. In: Proceedings of the 14th Swiss Transport Research Conference (2014)

[27] Martinez G., F.A.: Reinforcement learning in a multi-agent framework for pedestrian simulation. Ph.D. thesis, University of Valencia (2014)

[28] Chen, L.: Towards a multi-agent based software framework for fine-scale pedestrian movement modelling. International Journal of Modelling and Simulation 34(2), 5945 (2014). doi:10.2316/Journal.205.2014.2.205-5945

[29] Daniel, J., Truong, C., Lauffenburger, J., Basset, M.: Real-time trajectory generation for advanced driver assistance systems applications. In: International Forum On Strategic Technologies (IFOST09), vol. 26 (2009)

[30] Helbing, D., Farkas, I., Vicsek, T.: Simulating dynamical features of escape panic. Nature 407, 487-490 (2000). doi : 10 . $1038 / 35035023$

[31] Kretz, T.: On oscillations in the social force model. Physica A: Statistical Mechanics and its Applications 438, 272-285 (2015). doi:10.1016/j.physa.2015.07.002

[32] Johansson, A., Helbing, D., Shukla, P.: Specification of the social force pedestrian model by evolutionary adjustment to video tracking data. Advances in Complex Systems 10(supp02), 271-288 (2007). doi:10.1142/S 0219525907001355

[33] Seyfried, A., Steffen, B., Lippert, T.: Basics of modelling the pedestrian flow. Physica A: Statistical Mechanics and its Applications 368(1), 232-238 (2006). doi:10.1016/j.physa.2005.11.052

[34] Helbing, D., Molnar, P.: Social force model for pedestrian dynamics. Physical review E 51(5), 4282 (1995). doi:10.1103/PhysRevE.51.4282

[35] Wolfram-Alpha: (2015). Publisher: Wolfram Alpha LLC. Retrieved March 24th 2015. https://www.wolframalpha.com/input/?i=solve+\%282x$1 \div 29 * \exp \div 281 \div 2 \mathrm{Fx} \div 29+-+0.9 * \div 282 \times \div 2 \mathrm{~B} 1 \div 29+\mathrm{for}+\mathrm{x}$ 
[36] Yu, W., Chen, R., Dong, L., Dai, S.: Centrifugal force model for pedestrian dynamics. Physical Review E 72(2), 026112 (2005). doi:10.1103/PhysRevE.72.026112

[37] Yu, W., Johansson, A.: Modeling crowd turbulence by manyparticle simulations. Physical Review E 76(4), 046105 (2007). doi:10.1103/PhysRevE.76.046105

[38] Chraibi, M., Seyfried, A., Schadschneider, A.: Generalized centrifugal-force model for pedestrian dynamics. Physical Review E 82(4), 046111 (2010). doi:10.1103/PhysRevE.82.046111

[39] Campanella, M., Hoogendoorn, S., Daamen, W.: The nomad model: theory, developments and applications. Transportation Research Procedia 2, 462-467 (2014). doi:10.1016/j.trpro.2014.09.061

[40] Sherif, M.: The psychology of social norms. Harper (1936)

[41] Kretz, T., Große, A., Hengst, S., Kautzsch, L., Pohlmann, A., Vortisch, P.: Quickest Paths in Simulations of Pedestrians. Advances in Complex Systems 14, 733-759 (2011). doi:10.1142/S0219525911003281

[42] Kretz, T., Große, A.: From Unbalanced Initial Occupant Distribution to Balanced Exit Usage in a Simulation Model of Pedestrian Dynamics. In: T.J. Shields et al. (ed.) Human Behaviour in Fire Symposium, pp. 536-540. Interscience Communications (2012)

[43] Kretz, T.: Multi-Directional Flow as Touch-Stone to Assess Models of Pedestrian Dynamics. In: Annual Meeting of the TransportationResearch Board 2013 (2013). $13-1160$

[44] Kretz, T.: The Effect of Integrating Travel Time. In: Weidmann, U., Kirsch, U., Schreckenberg, M. (eds.) Pedestrian and Evacuation Dynamics 2012, pp. 10131027 (2013)

[45] Ziemer, V., Seyfried, A., Schadschneider, A.: Congestion dynamics in pedestrian single-file motion. In: Knoop, V., Daamen, W. (eds.) Traffic and Granular Flow 2015 (preprint)

[46] Zhang, J., Mehner, W., Andresen, E., Holl, S., Boltes, M., Schadschneider, A., Seyfried, A.: Comparative analysis of pedestrian, bicycle and car traffic moving in circuits. Procedia-Social and Behavioral Sciences 104, 1130-1138 (2013). doi:10.1016/j.sbspro.2013.11.209

[47] Seyfried, A., Zhang, J., Andresen, E., Boltes, M., Mehner, W., Holl, S., Schadschneider, A.: Universalities in fundamental diagrams of cars, bicycles and pedestrians. In: Symposium Celebrating 50 Years of Traffic Flow Theory, FZJ-2014-06019. Jülich Supercomputing Center (2014) 
[48] Sugiyama, Y., Fukui, M., Kikuchi, M., Hasebe, K., Nakayama, A., Nishinari, K., Tadaki, S., Yukawa, S.: Traffic jams without bottlenecks - experimental evidence for the physical mechanism of the formation of a jam. New Journal of Physics 10(3), 033001 (2008). doi:10.1088/1367-2630/10/3/033001

[49] Nakayama, A., Fukui, M., Kikuchi, M., Hasebe, K., Nishinari, K., Sugiyama, Y., Tadaki, S., Yukawa, S.: Metastability in the formation of an experimental traffic jam. New Journal of Physics 11(8), 083025 (2009). doi:10.1088/1367-2630/11/8/083025

[50] PTV AG: PTV Vissim 8.0 - User Manual. PTV Group, Haid-und-Neu-Str. 15, D76131 Karlsruhe, Germany (2015)

[51] Hupfer, C.: Computergestützte Videobildverarbeitung zur Verkehrssicherheitsarbeit - am Beispiel von Fußgängerquerungen an städtischen Hauptverkehrsstraßen. Ph.D. thesis, University of Kaiserslautern (1997)

[52] Zhang, J., Klingsch, W., Schadschneider, A., Seyfried, A.: Transitions in pedestrian fundamental diagrams of straight corridors and t-junctions. Journal of Statistical Mechanics: Theory and Experiment 2011(06), P06004 (2011). doi:10.1088/1742-5468/2011/06/P06004

[53] Lighthill, M., Whitham, G.: On kinematic waves. ii. a theory of traffic flow on long crowded roads. In: Proceedings of the Royal Society of London A: Mathematical, Physical and Engineering Sciences, vol. 229, pp. 317-345. The Royal Society (1955). doi:10.1098/rspa.1955.0089

[54] Heydecker, B., Addison, J.: Analysis and modelling of traffic flow under variable speed limits. Transportation Research Part C: Emerging Technologies 19(2), 206217 (2011). doi:10.1016/j.trc.2010.05.008

[55] Treiber, M., Kesting, A.: Traffic Flow Dynamics: Data, Models and Simulation. Springer-Verlag Berlin Heidelberg (2013). doi: $10.1007 / 978-3-642-32460-4$

[56] Takayasu, M., Takayasu, H.: 1/f noise in a traffic model. Fractals 1(04), 860-866 (1993). doi:10.1142/s0218348X93000885

[57] Schadschneider, A., Schreckenberg, M.: Traffic flow models with slow-to-start rules. Annalen der Physik 509(7), 541-551 (1997). 